\title{
TUMORES DE GLÁNDULA SUBMANDIBULAR: REPORTE DE UN CASO DE CARCINOMA ADENOIDE QUÍSTICO
}

Submandibular gland tumors: Report of a case of adenoid cystic carcinoma

Fecha de Recepción: 28 de agosto 2021
Tumores da glândula submandibular: Relato de um caso de carcinoma adenóide cístico

Aceptado para su publicación: 19 de octubre 2021
1. Departamento de Biología Bucal. Facultad de Odontología. Universidad Nacional de Córdoba.

2. Cátedra de Biología Celular, Histología y Embriología. Facultad de Ciencias Médicas. Universidad Nacional de Córdoba.

3. Cátedra de Anatomía. Facultad de Ciencias Médicas. Universidad Nacional de Córdoba. 4. II Cátedra de Patología. Facultad de Ciencias Médicas. Universidad Nacional de Córdoba. a. Doctor en Medicina y Cirugía (Universidad Nacional de Córdoba)

Correspondencia: Samar Romani, María Elena Catamarca 1546. Córdoba (5000). Argentina $+5403514510182$

Correo electrónico: maría.elena.samar@unc.edu.ar samarcongreso@gmail.com

Conflicto de intereses: los autores declaran no tener conflictos de interés.

Fuente de financiamiento: Subsidio CONSOLIDAR. Secretaría de Ciencia y Tecnología 05/H575 período 2018-2022. Universidad Nacional de Córdoba. Argentina. Programa de incentivos.

\section{Resumen}

Los tumores de la glándula submandibular son poco comunes, con tipos histológicos diversos y pronóstico variable. El propósito de este estudio fue presentar un caso de carcinoma adenoide quístico submandibular en una paciente de 54 años y analizar sus características histológicas para determinar su grado de malignidad y el tratamiento a seguir. El patrón sólido que predomina en el caso presentado tiene un riesgo mayor de metástasis a distancia. Resultan clave el diagnóstico y la atención oncológica precoces para su mayor posibilidad de curación y sobrevida.

Palabras clave: glándula submandibular; neoplasias; carcinoma adenoide quístico (fuente: DeCS BIREME).

\section{Abstract \\ Tumors of the submandibular gland are rare, with diver- se histological types and variable prognosis. The purpo- se of this study was to present a case of submandibular adenoid cystic adenoid carcinoma in a 54-year-old fe- male patient and to analyze its histological features to determine its degree of malignancy and further treat- ment. The solid pattern that predominates in the pre- sented case has a higher risk of distant metastasis. Early diagnosis and oncological care are key to a better chan- ce of cure and survival.}

Key words: submandibular gland; neoplasms; carcinoma, adenoid cystic (source: MeSH NLM).

\section{Resumo}

Os tumores da glândula submandibular são raros, com diversos tipos histológicos e prognóstico variável. O objetivo deste estudo é apresentar um caso de carcinoma adenóide cístico submandibular em paciente de 54 anos e analisar suas características histológicas para 
determinar seu grau de malignidade e o tratamento a ser seguido. O padrão sólido que predomina no caso apresentado apresenta maior risco de metástases à distância. O diagnóstico precoce e os cuidados oncológicos são fundamentais por sua maior chance de cura e sobrevivência.

Palavras-chave: glândula submandibular; neoplasias; carcinoma adenóide cístico (fonte: DeCS BIREME).

\section{Introducción}

Como destacan Gontarz et al. ${ }^{1}$ los tumores de las glándulas salivales son neoplasias infrecuentes que comprenden aproximadamente 3 a $6 \%$ de toda la patología neoplásica de cabeza y cuello. La localización anatómica más observada es la glándula parótida, seguida por la submandibular y las glándulas salivales menores. En ciertos casos, estas lesiones también se originan en el tracto aerodigestivo superior y las glándulas lagrimales ${ }^{2-4}$.

Existe considerable literatura sobre las neoplasias salivales en general, pero sólo escasos trabajos describen los tumores de la glándula submandibular. Estos son relativamente poco comunes, con tipos histológicos diversos y pronóstico variable va-7 $^{5 a-}$ jappa et al. ${ }^{8}$, mencionan que comprenden cerca de 8 a $9 \%$ de las neoplasias salivales y Sahin et al. ${ }^{7}$, un 5 a $15 \%$, siendo superior la proporción de tumores malignos relacionados con los benignos en glándula submandibular cuando se los compara con los de la parótida.

El tumor benigno más frecuente diagnosticado en submandibular es el adenoma pleomórfico y los malignos más comunes son el carcinoma adenoide quístico (CAQ), el carcinoma mucoepidermoide, el carcinoma de células escamosas y el adenocarcinoma ${ }^{8}$. Estos autores consideran que, además de ser infrecuentes en esta glándula, son muy heterogéneos en cuanto a su tipo histológico, presentación, recurrencia y sobrevida. Sahin et al. ${ }^{7}$, citan a Bradley et al. (2013) y Mariano et al. (2011), quienes demostraron una prevalencia de metástasis a distancia superior en los tumores localizados en submandibular cuando se los comparó con los de parótida.

En el presente trabajo presentamos un caso de CAQ de glándula submandibular izquierda, donde analizamos su patrón morfológico para determinar su grado de malignidad y el tratamiento a seguir.

\section{Caso clínico}

Paciente femenina de 54 años que consultó por una tumoración localizada por debajo de la mandíbula izquierda, de 6 meses de evolución, con dolor a la palpación y un crecimiento progresivo el último mes (Figura 1A). Al examen físico medía $5 \mathrm{~cm}$ de diámetro, bien delimitada, dura y fija a las estructuras vecinas, con la piel de la zona sin particularidades. No se palparon nodos linfoides satélites.

Se indicó una tomografía axial computarizada del cuello, informándose la presencia de una masa sólida y heterogénea, de $5,5 \mathrm{~cm}$ de diámetro, que ocupaba la región submandibular izquierda (Figura 1B, CyD).

Se efectuó la resección realizándose una incisión arciforme, a 3-4 cm por debajo del ángulo mandibular sobre el borde anterior del músculo esternocleidomastoideo, hacia abajo y adelante hasta el nivel del asta menor del hueso hioides. Se ligó y seccionó la vena facial posterior, traccionando el cabo superior hacia arriba para proteger la rama marginal del nervio facial, que cruza por la cara lateral de este vaso. Se ligó y seccionó la arteria facial y luego se resecó el nódulo que formaba parte de la glándula submandibular, respetando el nervio lingual.

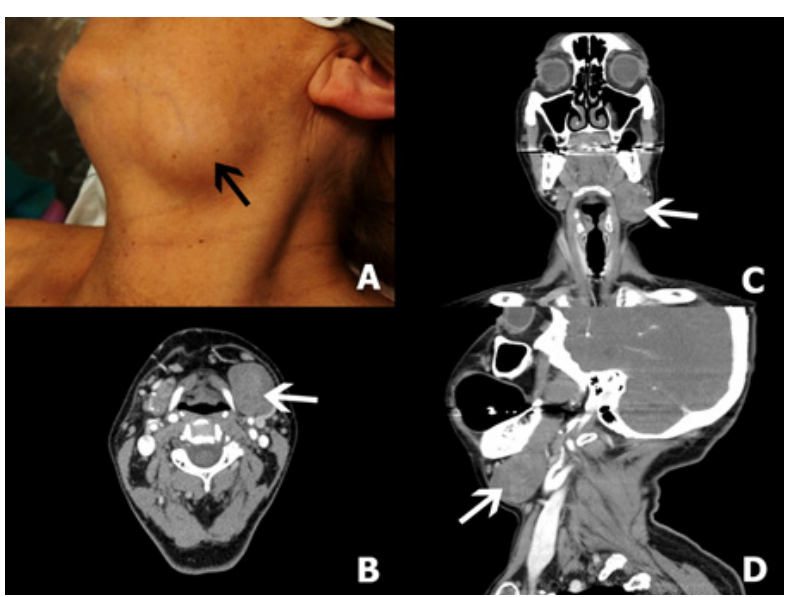

Figura 1. Carcinoma adenoide quístico de glándula submandibular. A. Se señala la localización de la tumoración motivo de la consulta clínica. B. Tomografía axial computarizada. Corte axial. C. Tomografía axial computarizada. Corte coronal. D. Tomografía axial computarizada. Corte sagital. 


\section{REFO}

VOL. XIV | N² 2| AÑO 2021

ISSN 1668-7280

ISSN-E 2683-7986
El diagnóstico histopatológico de los cortes de la pieza quirúrgica coloreados con Hematoxilina y eosina y Tricrómico de Dane fue CAQ, donde coexistían tres patrones morfológicos: cribiforme, tubular y sólido, comprendiendo este último más del $30 \%$ del tumor, característico de una lesión Grado III de malignidad (Figura 2A, B y C). Las estructuras cribiformes estaban constituidas por islotes celulares con espacios seudoquísticos y seudoglandulares de tamaño heterogéneo. El patrón tubular presentó estructuras epiteliales de apariencia ductal, que en ocasiones contenían un material luminal débilmente eosinofílico. Cordones densamente celulares caracterizaban al componente sólido. Se observó, además, invasión perineural (Figura 3).

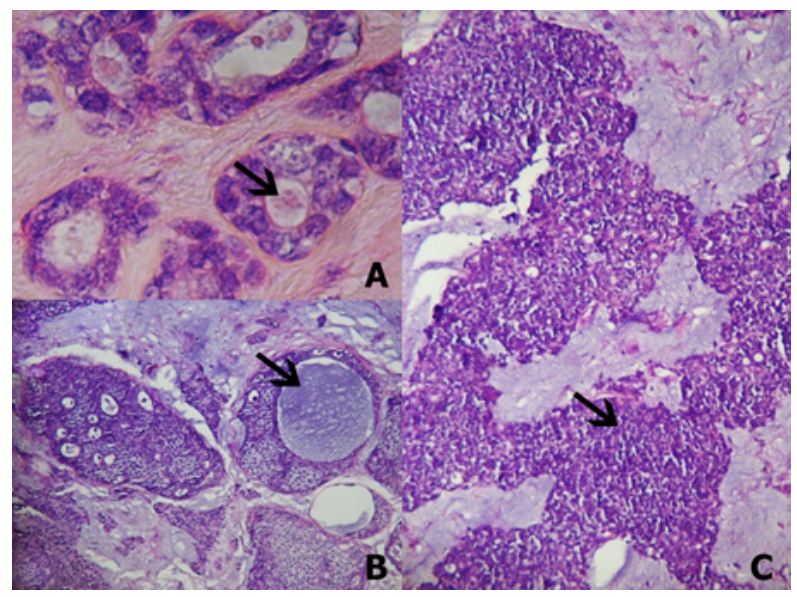

Figura 2. Carcinoma adenoide quístico de glándula submandibular. A. Patrón tubular. Estructuras epiteliales de apariencia ductal (flecha). Coloración H/E. Objetivo 40x. B. Patrón cribiforme. Islotes celulares atravesados por espacios seudoquísticos y seudoglandulares (flecha). Coloración H/E. Objetivo 40x. C. Patrón sólido. Cordones densamente celulares (flecha). Coloración H/E. Objetivo 40x.

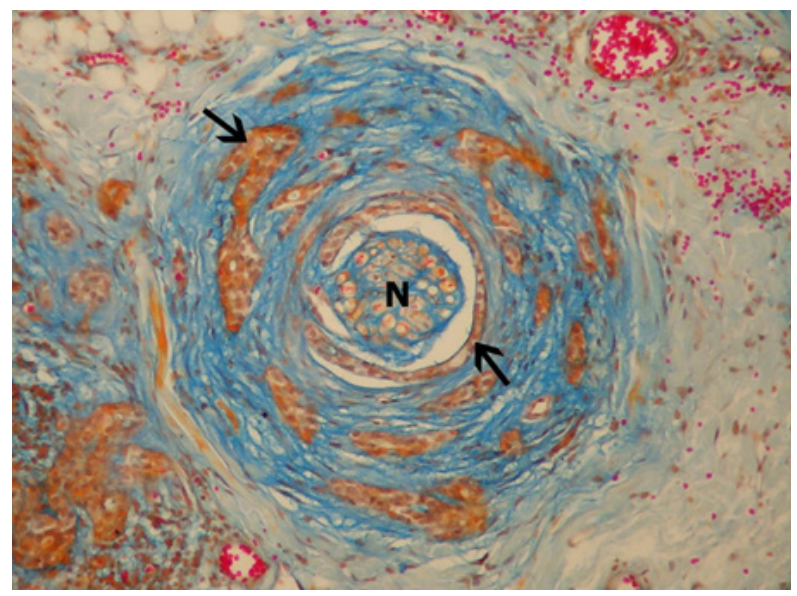

Figura 3. Carcinoma adenoide quístico de glándula submandibular. Se señala la invasión perineural del tumor (flecha). Nervio (N): Coloración Tricrómico de Dane. Objetivo 20X.
El predominio del componente sólido y la invasión perineural se consideraron factores de riesgo por lo que se indicó radioterapia postoperatoria, iniciada a los 40 días de la cirugía. La paciente se recuperó satisfactoriamente, sin secuelas en los nervios facial y lingual. En la TAC de control de cuello y tórax, no se observó en la actualidad extensión pulmonar o cervical. Se debe seguir el monitoreo periódico permanente de la paciente.

\section{Discusión}

Diferentes autores vinculan a los tumores de la glándula submandibular con un mayor índice de malignidad y un peor pronóstico en relación con los otros tumores salivales, especialmente de la paróti$\mathrm{da}^{9,10}$. Por otro lado, el CAQ (Código ICD-O 8200/3) es el tumor maligno que se desarrolla más frecuentemente en las glándulas salivales menores y en las glándulas salivales mayores, principalmente submandibular 2,3,5,11. También compromete a las glándulas lagrimales y ceruminosas y otras localizaciones en cabeza y cuello.

Este tumor epitelial maligno es indolente pero agresivo, con crecimiento lento y destructivo, constituido por células con diferenciación ductal y mioepitelial modificadas. Se diferencia de otros tumores de glándulas salivales de similar composición celular, por sus características citomorfológicas y un patrón de crecimiento cribiforme, tubular y sólido ${ }^{12,13}$. Según el patrón de crecimiento que predomina se lo clasifica en Grados I, II y III, como se describe en el clásico trabajo de Perzin et al. ${ }^{14}$. EI CAQ Grado I desarrolla componentes cribiforme y tubular, el tumor Grado II componentes cribiforme, tubular y menos del $30 \%$ de sólido y el Grado III componentes cribiforme, tubular y más de $30 \%$ de sólido ${ }^{13,14}$.

En el caso presentado en este trabajo, la paciente desarrolló un CAQ de glándula submandibular izquierda cuya histopatología mostró una lesión predominantemente sólida. El patrón sólido se asocia a recurrencias más frecuentes y a una mayor ocurrencia de metástasis. Perzin et al. ${ }^{14}$, Ben Salha et al. ${ }^{15} \mathrm{y}$ Szanto et al. ${ }^{16}$ consideran que este componente se asocia a un peor pronóstico cuando se desarrolla en una proporción superior al 30\%. 


\section{9}

CASO CLÍNICO

Tumores de Glándula submandibular: Reporte de un caso de carcinomaadenoide quístico.
REFO

VOL. XIV | N² 2| AÑO 2021

ISSN 1668-7280

ISSN-E 2683-7986
Además, este tumor produjo en nuestra paciente invasión perineural, factor pronóstico importante en tumores salivales malignos determinado por la presencia de epiteliocitos tumorales dentro del espacio perineural, espacio vacío entre los nervios periféricos y las células perineurales y que incrementa el desarrollo de metástasis y la invasión tumoral, afectando la sobrevidaa ${ }^{17,18}$. No obstante, existe un debate en cuanto a la asociación entre la invasión perineural y las metástasis sistémicas ${ }^{19}$. Dantas et al. ${ }^{20}$ realizaron una búsqueda sistemática en las bases de datos PubMed/MEDLINE, SciElo, Science Direct and Scopus de artículos publicados entre junio de 2000 y junio de 2014 y concluyeron que la invasión perineural no se asocia con metástasis o tasa de supervivencia sino con la presencia de recurrencia local y el compromiso de los márgenes quirúrgicos.

Por otro lado, varios estudios clínicos indican que los tumores malignos localizados en la glándula submandibular se relacionan con mayor número de metástasis, a diferencia de lo que ocurre con aquéllos originados en la parótida. Shin et al. ${ }^{21}$ consideran que el microambiente de la glándula submandibular tiene mayor abundancia de vasos sanguíneos asociados al tumor, a diferencia de lo que ocurre en el CAQ de parótida, lo cual explicaría el riesgo incrementado de las metástasis a distancia del tumor submandibular. Sin embargo, se deben profundizar estudios de los mecanismos de angiogénesis para arribar a conclusiones más evidentes.
El tratamiento del CAQ depende de su localización, estadio al momento de realizar su diagnóstico y su comportamiento biológico, relacionado con su histología, que indica el grado tumoral. Coca-Pelaz et al. ${ }^{11}$ consideran que el método elegido para tratar el CAQ es la resección quirúrgica radical asegurando márgenes libres, acompañada de radioterapia postoperatoria. Se ha comparado la radioterapia sola con la combinación cirugía/radioterapia y Mendenhall et al. ${ }^{22}$ concluyen también que esta última debe ser el tratamiento de elección.

Kim et al. ${ }^{23}$ destacan que el cáncer primario de parótida también ofrece dificultades cuando se debe decidir una conducta terapéutica debido igualmente a su diferente potencial maligno y a su heterogénea histología. Consideran que la cirugía es el tratamiento principal y se debe completar con radioterapia postoperatoria en pacientes con tumores con márgenes positivos, alto grado de malignidad histológica, invasión perineural/vascular/linfática y nodos linfoides metastásicos.

Se concluye que el patrón sólido, que predomina en el caso presentado y la invasión perineural tienen un riesgo mayor de recurrencias, metástasis a distancia y una peor sobrevida. Algunos estudios indican que el pronóstico de los pacientes con cáncer de la glándula submandibular es peor que el de parótida y con mayor incidencia de metástasis ocultas. Resultan clave el diagnóstico y la atención oncológica precoces con un control y seguimiento periódicos de la paciente que dan mayor posibilidad de curación y sobrevida. 


\section{Referencias Bibliográficas}

1. Gontarz M, Bargiel J, Gąsiorowski K, Marecik T, Szczurowski P. Zapala J. Epidemiology of primary epithelial salivary gland tumors in Southern Poland -A 26-year, clinicopathologic, retrospective analysis. J Clin Med. 2021;10(8),1663. doi: 10.3390/jcm10081663

2. Avila RE, Samar ME, Corball AG, Fonseca IB, Ferraris RV. Carcinoma adenoide quístico extrasalival. Presentación de un caso. Rev Esp Patol. 2010;43(2):126-128. doi: 10.1016/j.patol.2010.02.008

3. Samar ME, Avila RE, Corball A, Giraudo P. Carcinoma adenoide quístico invasor de cavidad nasal izquierda: una rara localización. Rev Fac Odont. 2018; 28(1):17-22. Disponible en: https://revistas.unc.edu.ar/index.php/RevFacOdonto/article/view/19760/19512

4. Gomez Rosso MA, Samar ME, Ávila RE, Ferraris L, Fonseca IB, Fernández JE. Adenoma pleomórfico de glándula lagrimal: localización atípica de un tumor salival. Rev Fac Odont. 2020;30(3):20-28. doi: 10.25014/revfacodont271.2020.30.3.20

5. Avila RE, Samar ME, Ferraris R, La Rosa F. Oncocytic mucoepidermoid carcinoma of the submandibular salivary gland: a challenging differential diagnosis of a very rare tumor. Ann Clin Cases Rep. 2019;4,1726. Disponible en: https://www.researchgate.net/publication/337873275

6. Coupland A, Sewpaul A, Darne A, White S. Adenoid cystic carcinoma of the submandibular gland, locoregional recurrence, and a solitary liver metastasis more than 30 years since primary diagnosis. Case Rep Surg. 2014; 2014:581823. doi: 10.1155/2014/581823

7. Sahin MM, Demircan V, Göcek M, Uzunoğlu E, Akmansu M, Sayar E, et al. Submandibular gland cancers: prognostic factors and survival analysis. Eur Arch Otorhinolaryngol. 2020; 277(8):2307-2313. doi: 10.1007/s00405-02005924-w

8. Rajappa SK, Bhakuni YS, Ram D, Shukla H, Ranjan R, Dewan A, Dabas S, Dewan AK. A gland of diverse pathology and unpredictable behaviour: our experience of primary submandibular gland malignancies. Int J Oral Maxillofac Surg. 2018; 47(10):1243-1249, doi: 10.1016/j. ijom.2018.05.012
9. Lee RJ, Tan AP, Tong EL, Satyadev N, Christensen RE. Epidemiology, prognosis factors and treatment of malignant submandibular gland tumors. A population-based cohort analysis. JAMA Otolaryngol Head Neck Surg. 2015; 141(10): 905-912. doi: 10.1001/jamaoto.2015.1745

10. Aro K, Tarkkanen J, Saat R, Saarilahti K, Mäkitie A, Atula T. Submandibular gland cancer: Specific features and treatment considerations. Head Neck. 2018;40(1): 154162. doi: 10.1002/head.24981

11. Coca-Pelaz A, Rodrigo JP, Bradley PJ, Vander Poorten V, Triantafyllou A, Hunt JL, et al. Adenoid cystic carcinoma of the head and neck. An update. Oral Oncol. 2015; 51(7): 652-.661. doi: 10.1016/j.oraloncology.2015.04.005

12. Cantú G. Adenoid cystic carcinoma. An indolent but aggressive tumour. Part A: from aetiopathogenesis to diagnosis. Acta Otorhinolaryngol Ital. 2021; 41(3): 206214. doi.org/10.14639/0392-100X-N1379

13. Samar ME, Avila RE, Fernández JE. Glosario de Histopatología: tumores epiteliales de las glándulas salivales. 5a ed. ampliada. Samar ediciones. Córdoba. 2020.

14. Perzin KH, Gullane P, Clairmont AC. Adenoid cystic carcinoma arising in salivary glands: A correlation of histologic features and clinical course. Cancer. 1978; 42: 265-282. doi: 10.1002/1097-0142(197807)42:1 <265::aid-cncr2820420141>3.0.co;2-z.

15. Ben Salha I, Bhide S, Mourtzoukou D, Fisher C, Thway K. Solid variant of adenoid cystic carcinoma: Difficulties in diagnostic recognition. Int J Surg Pathol. 2016; 24(5): 419-424. doi: 10.1177/1066896916642011

16. Szanto PA, Luna MA, Tortoledo ME, White RA. Histologic grading of adenoid cystic carcinoma of the salivary gland. Cancer. 1984; 54(6): 1062-1069. doi: 10.1002/1097-0142(19840915)54:6<1062::aid-cncr2820540622>3.0.co;2-e

17. Chen SH, Zhang BY, Zhou B, Zhu CZ, Sun Q, Feng YJ. Perineural invasion of cancer: a complex crosstalk between cells and molecules in the perineural niche. Am Cancer Res. 2019;9(1):1-21. Disponible en: https://www. ncbi.nlm.nih.gov/pmc/articles/PMC6356921/ 
18. Cohen AN, Damrose EJ, Huang RY, Nelson SD, Blackwell KE, Calcaterra TC. Adenoid cystic carcinoma of the submandibular gland: A 35-year review. Otolaryngol Head Neck Surg. 2004; 131(6): 994-1000. doi: 10.1016/j. otohns.2004.06.705

19. Lukšić I, Baranović S, Suton P, Gerbl D. Adenoid cystic carcinoma of the head and neck: a single institution's analysis of 45 consecutive cases over a 29-year period. Oral Surg Oral Med Oral Pathol Oral Radiol. 2016; 122(2): 152-157. doi: 10.1016/j.00oo.2016.03.007

20. Dantas AN, Freitas de Morais E, de Paiva Macedo RA, de Lima Tinôco JM, Silva de Arruda Morais M de L. Clinicopathological characteristics and perineural invasion in adenoid cystic carcinoma: a systematic review. Braz J Otorhinolaryngol. 2015; 81(3): 329-335. doi: 10.1016/j. bjorl.2014.07.01

21. Shin DY, Jang KS, Kim BY, Choi JE, Yoon H, Ko YH, Jeong HS. Comparison of adenoid cystic carcinomas arising from the parotid gland vs. the submandibular gland: focus on systemic metastasis and tumor-associated blood vessels. J Oral Pathol Med. 2014;43(6):441-7. doi: 10.1111/jop.12155

22. Mendenhall WM, Morris CG, Amdur RJ, Werning JW, Hinerman RW, Villaret DB. Radiotherapy alone or combined with surgery for adenoid cystic carcinoma of the head and neck. Head Neck. 2004;26(2): 154-162. doi: 10.1002/ hed. 10380

23. Kim YH, Chung WK, Jeong JU, Cho IJ, Yoon MS, Song JY et al. Clin Exp Otorhinolaryngol. 2020;13(1):69-76. doi: $10.21053 /$ ceo.2019.00388
Cumplimientos de estándares éticos: Este trabajo es parte del proyecto "La matriz extracelular y el componente mioepitelial de los tumores epiteliales de glándulas salivales humanas: estudio estructural, histoquímico e inmunohistoquímico" aprobado por el Comité de Ética del Hospital Nacional de Clínicas. Universidad Nacional de Córdoba. Argentina (Registro 188/14). 